\title{
EXPERIMENTAL ANALYSIS OF MATCHING TECHNIQUE OF STEGANOGRAPHY FOR GREYSCALE AND COLOUR IMAGE
}

\author{
Khurrum Rahim Rashid ${ }^{\# 1}$, Aqsa Rashid ${ }^{* 2}$, Nadeem Salamat ${ }^{\# 3}$ and Saad Missen ${ }^{\# 4}$ \\ ${ }^{\# 1}$ Department of Electrical Engineering, NUSES FAST, Pakistan \\ ${ }^{\# 2,4}$ Department of CS\&IT, Islamia University Bahawalpur, Pakistan \\ ${ }^{\# 3}$ Department of Mathematics \& Statistics, Karakoram International University Gilgit, \\ Gilgit-Biltastan
}

\begin{abstract}
Steganography and steganalysis are vital matter in information hiding. Steganography refers to the technology of hiding data into digital media without depiction of any misgiving, while steganalysis is the detection of the existence of steganography. In this paper, the impact of sequential matching method for every possible location in pixel is analyzed experimentally. After matching the steganalysis is check by image quality measures (IQM) and Statistics, Histogram Analysis and visual attack. This analysis provides comprehensive study and understanding of basic matching technique and is helpful for those who want to work in the field of image steganography.
\end{abstract}

\section{KEYWORDS}

Stagnography, Least significant bit, Matching, Statistical trial for comparison.

\section{INTRODUCTION}

Steganography has established significant attention during the last decade, in particular after anecdotal news suspected that this tool was used by terrorist. Steganography $[2,3]$ inquire about to make available a clandestine message control between two parties. The data that are passing on through internet persistently have possibility of third individual nosy. So there should be some criteria to keep information covert today as the current time is of digital contact. There are various steganography technique and medium used for this purpose [1][4][5][8]. These methods are gaining value due to the secret communication over the internet.

In sequential matching method, the pixel value is incremented or decremented to match the message and pixel bit. The process of matching keep on until the length of the message bits becomes zero. This can be applied on both the greyscale and colour image.

The paper is arranged as Section II describes the method of matching, Section III discuss the steganalysis, section IV gives experimental results and discussion, section V describes conclusion

\section{METHOD}

Matching steps for matching LSB Stego-method are: 


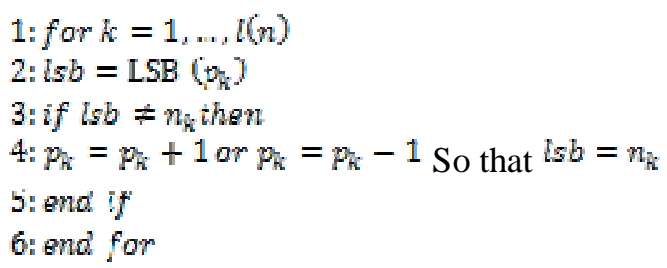

(in) Contain the message bits. It first takes the pixel $p_{k}$ of the image and its $\operatorname{LSB}\left(p_{k}\right)$ value. If matching bit and LSE $\left(p_{k}\right)$ are now the same, then nothing to be done, but if they are dissimilar then increment or decrement in $p_{k i}$ in such a way that the LSB ( $p_{h}$ ) become the matching bit. This process goes on even as $l(n)$ is not zero.

Extracting steps for sequential matching Stego-method

1: for $k=1, \ldots, l(t)$

2: $r m_{k}-\left(t_{k}\right)$

3 end for

The $l(t)$ is the total number of pixels of supposed image. Run the loop $[(t)$ in place of $l(n)$. This is because the embedding is different from the retrieval process. We just recover the LSB value of each pixel in ${ }^{\mathrm{mm}}$ and translate this to ASCII; the message will be understandable and in readable format up to the point that the message was embedded, and will then come into view as claptrap when we are see the LSBs of the image data. If we know the length of the message that was embedded, then the loop will be ended when the length of message is completed and only the message will be retrieved i.e., no gibberish will be seen at the end of the message.

\section{STEGANALYSIS}

This part deals with the steganalysis of the matching techniques. It is divided into three subsections. First deals with Steganalysis by Image Quality measures [6], Histogram, second deals with Steganalysis by Histogram Analysis and third is Steganalysis by Bit Planes Analysis (Visual Attack).

\section{Image Quality Measure}

In the paper four most important and widely used Image quality measures $[7,9,10,11,12]$ namely MSE, PSNR, UIQI and SSIM are computed for steganalysis. Mean Square Error (MSE) computes the perceived error. It is pixel value difference based quality measure. Peak Signal to Noise Ratio (PSNR) [10] is inversely proportional to MSE. Less MSE gives High PSNR which is the proof of the fact that image has good quality.

Universal Image Quality Index split the judgment of similarity between Cover Image (CI) and Stego-Image (SI) into three comparisons: Luminance, Contrast and Structural Information.

SSIM estimates "Perceived change in structural information". It computes the similarity between two images of common size. Its mathematical definition is as:

The value of UIQI and SSIM varies between 1 and -1 . Closer the highest positive value denotes too much less change in two images and -1 shows totally mismatch. The UIQI and SSIM are considered as more consistent and accurate than MSE and PSNR. 


\section{A. Histogram Analysis}

Each bin of the histogram represents the number of pixels, which have the value represented by that bin. After LSB matching the local maxima of the histogram of images will decrease and the local minima will increase. Figure 1 (a) is the histogram of cover image and (b) shows the histogram of stego-image.

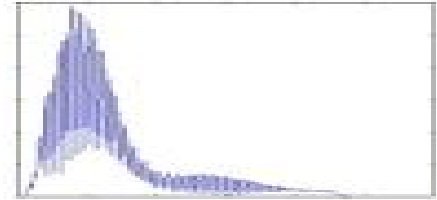

call

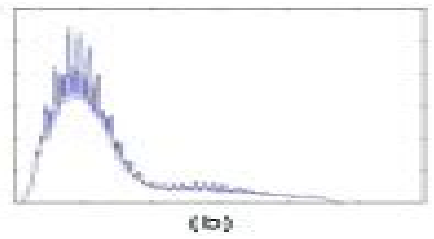

Fig.1 (a) Histogram of Cover Image (b) Histogram of Stego-image

For histogram analysis Jaccard measure, Correlation, Chi-square, Intersection and Bhattacharya distance [6] are computed between the histogram of cover image and stego-image. All these comparisons are performed on normalized histogram. The correlation value varies between 1 and -1 . Perfect match is 1 and total mismatch is -1 . For Chi-square ideal value is 0 and mismatch value is unbound, for intersection 1 is ideal matching value and 0 is mismatched value and Bhattacharya distance gives 0 for the exact match and 1 for mismatch. When these comparison matrices gives ideal values or values that are closer to ideal values then the change in histogram is very least and this is the evidence for Stego-System to be a secure system.

\section{B. Visual Attack}

Figure 2 shows the concept of bit planes for the greyscale images. In image steganography visual hit distinguish whether or not a hypothetical image has been subjected to LSB matching steganography, the steganalyst will be looking to obtain a visual inconsistency that will successfully point out signs of embedding. Figure 3 shows the possibility of matching sequence and effect of matching sequence on the bit plan.
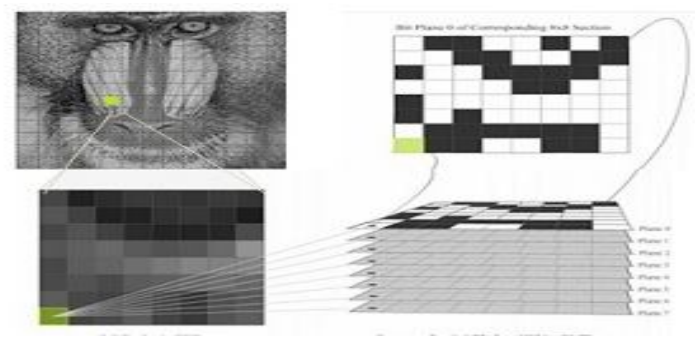

Fig.2 Bit Plan Concept

\section{EXPERIMENTAL RESULTS AND DISCUSSION}

The first part of this section includes the experimental results and discussion for the greyscale image and second part extend it for colour images.

Greyscale Images: First of all, we compare the perceptual impact of the image after hiding secret information. Secondly, impact on the image by statistical measures is compared. Then histogram analysis is performed and finally, visual attack by inspecting all bit plans of image is 
performed. First of all, experiments are performed for the greyscale images. In the next example, we extend our results for the colour images.

Fig 3 (a) is the original greyscale image, (b) is the histogram of the greyscale cover image that shows the minimum and maximum pixel value respectively, present in cover image and number of pixels having same colour and (c-j) are the bit plans of Cover image. Table I shows the results of Steganalysis by IQM. Table II shows the results of Histogram analysis. Fig 5 shows the bit plans of 0th (most significant bit MSB) to 7th (least significant bit LSB) bit stegoimages.
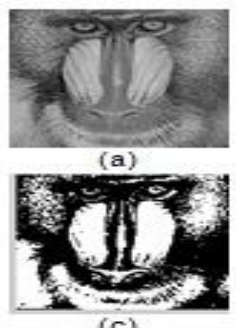

(c)

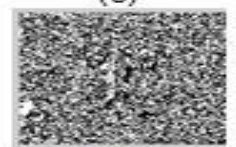

(g)

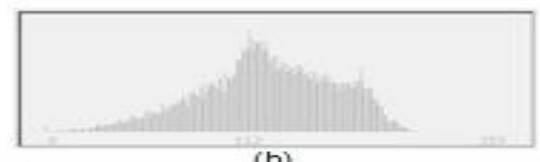

(b)

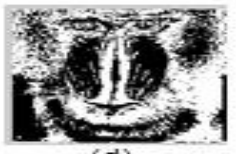

(d)

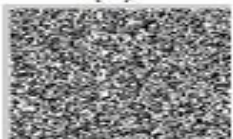

(h)

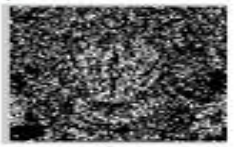

(e)

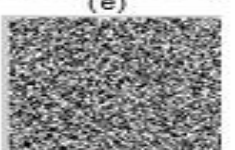

(i)

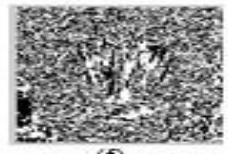

(f)

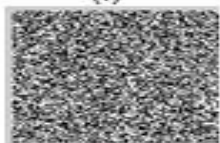

(j)

Fig 3 (a) Cover Image (b) Histogram of Cover Image (c-j) Bit plan of Cover Image

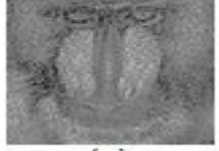

(a)

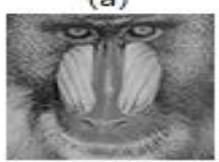

(e)

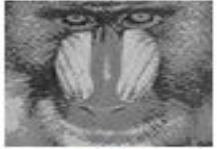

(b)

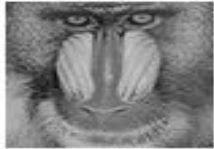

(f)

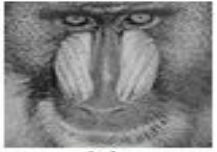

(c)

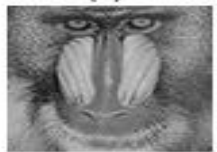

(g)

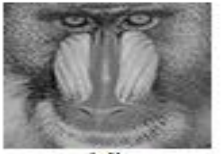

(d)

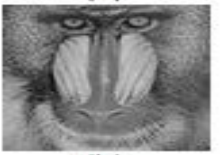

(h)

Fig 4 (a-h) $0^{\text {th }}(\mathrm{MSB})$ to $7^{\text {th }}$ (LSB) Bit Stego-Images

TABLE I Result of iqm for Gray scale Images

\begin{tabular}{|c|c|c|c|c|c|c|c|c|}
\hline QM & $\mathbf{0}^{\text {th }} \mathbf{B i t}$ & $\mathbf{1}^{\text {st }} \mathbf{B i t}$ & $\mathbf{2}^{\text {nd }} \mathbf{B i t}$ & $\mathbf{3}^{\text {rd }} \mathbf{B i t}$ & $\mathbf{4}^{\text {th }} \mathbf{B i t}$ & $\mathbf{5}^{\text {th }} \mathbf{B i t}$ & $\mathbf{6}^{\text {th }} \mathbf{B i t}$ & $\mathbf{7}^{\text {th }} \mathbf{B i t}$ \\
\hline MSE & $\begin{array}{c}676.3378 \\
181.9650 \\
9\end{array}$ & 50.76132 & $\begin{array}{c}12.6359 \\
8\end{array}$ & 3.77189 & 1.21497 & 0.49875 & 0.49449 \\
\hline PSNR & 19.83888 & 25.53092 & 31.075476 & 2 & 42.36521 & 4 & 51.15346 & 51.18917 \\
\hline NCC & 0.96769 & 0.99999 & 0.99999 & 0.99999 & 0.99999 & 1 & 1 & 1 \\
\hline UIQI & 0.66737 & 0.93663 & 0.98135 & 0.99529 & 0.99859 & 0.99955 & 0.99964 & 0.999815 \\
\hline SSIM & 0.66612 & 0.93646 & 0.98129 & 0.99529 & 0.99859 & 0.99955 & 0.99981 & 0.99992 \\
\hline
\end{tabular}


TABLE II RESULT OF HISTOGRAM COMPARISON FOR GREYSCALE IMAGES

\begin{tabular}{|l|c|r|r|r|r|r|r|r|}
\hline H-comp & $\mathbf{0}^{\text {th }} \mathbf{B i t}$ & $\mathbf{1}^{\text {st }} \mathbf{B i t}$ & $\mathbf{2}^{\text {nd }} \mathbf{B i t}$ & $\mathbf{3}^{\text {rd }} \mathbf{B i t}$ & $\mathbf{4}^{\text {th }} \mathbf{B i t}$ & $\mathbf{5}^{\text {th }} \mathbf{B i t}$ & $\mathbf{6}^{\text {th }} \mathbf{B i t}$ & $\mathbf{7}^{\text {th }} \mathbf{B i t}$ \\
\hline Jaccard & 0.95871 & 0.98901 & 0.99688 & 2 & 0.99977 & 0.99993 & 0.99997 & 0.99997 \\
\hline Intersection & 0.94437 & 0.97109 & 0.98207 & 4 & 0.99485 & 0.99698 & 0.99797 & 0.99776 \\
\hline Correlation & 0.70408 & 0.93818 & 0.98142 & $\begin{array}{r}0.9909 \\
9\end{array}$ & 0.99859 & 0.99955 & 0.99981 & 0.99981 \\
\hline Chi-square & 2.75833 & 0.74162 & 0.20791 & $\begin{aligned} 0.0517 \\
7\end{aligned}$ & 0.01545 & 0.00498 & 0.00203 & 0.00204 \\
\hline Bhattachary & & & & 0.0121 & & & & \\
a & 0.08158 & 0.04345 & 0.02321 & 4 & 0.00694 & 0.00415 & 0.00277 & 0.00250 \\
\hline
\end{tabular}

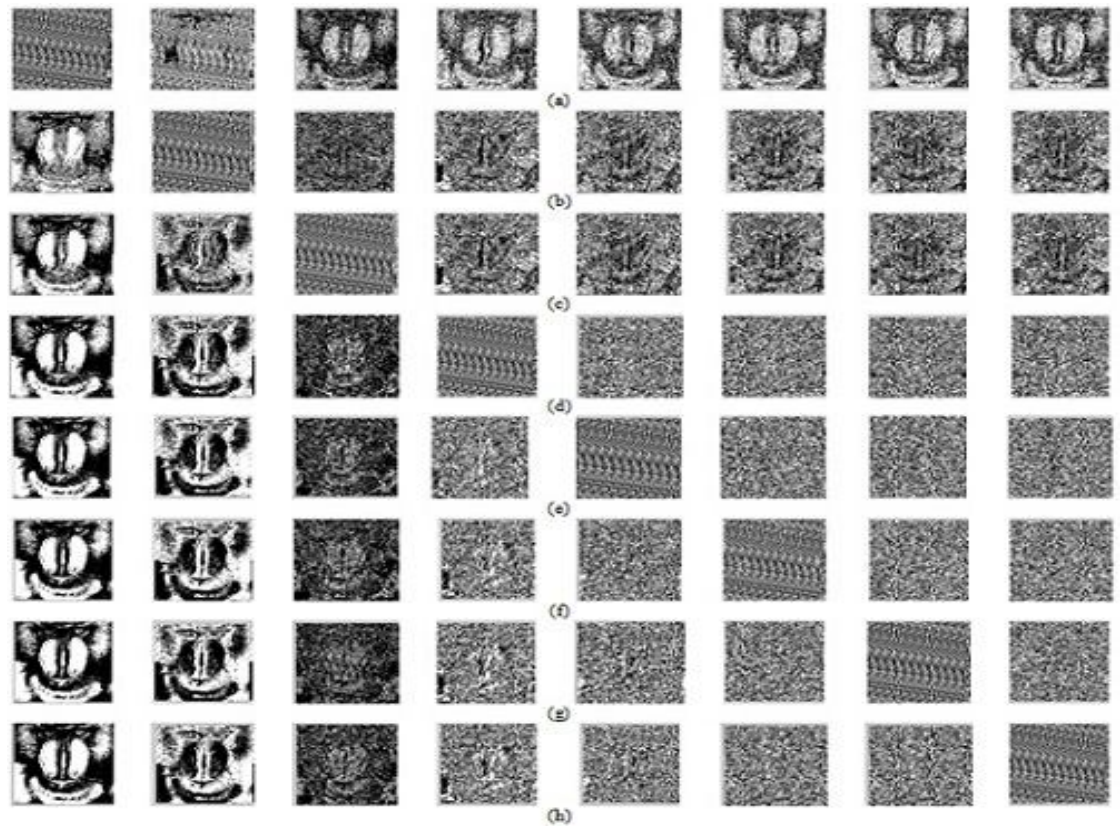

Figure 5 ( a-h) Bit plans of $0^{\text {th }}$ to $7^{\text {th }}$ Bit Stego-image

Colour Image: This section is extension of experimental results and discussion to colour images. Fig 6 (a) is the original RGB cover image having 34,645 pixels in pixel data (b) is the histogram of the RGB cover image that shows the minimum pixel value, maximum pixel value present in cover image and maximum number of pixels having same colour.(c),(d) and (e) are the channel based histogram of the RGB cover image. All the experimental results for RGB images are shown after replacing 96064 bits of RGB cover image.Fig.7 (a-c) shows the RGB bit plan of colour image of Fig 6 (a).

Fig $8(\mathrm{a}-\mathrm{h})$ are the $0^{\text {th }}$ to $7^{\text {th }}$ bit stego-images. Fig 9 to Fig 16 is the $0^{\text {th }}$ to $7^{\text {th }}$ bit RGB Bit plans of stego-images. Table III shows the result of IQM for colour image. Table IV shows the result of histogram comparison. 


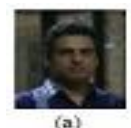

(a)

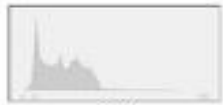

(b)

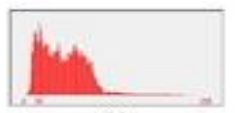

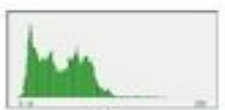

(d)

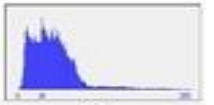

Fig 6 (a) Cover image (b) Histogram of Cover image(c-e) Channel Based Histogram of Cover image
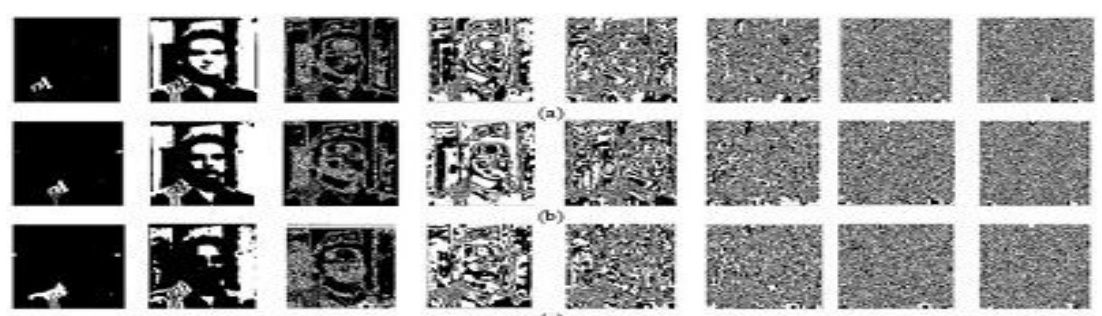

Fig 7 (a-c) Bit plans of RGB channel of Cover Image

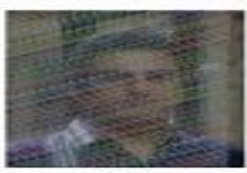

(a)

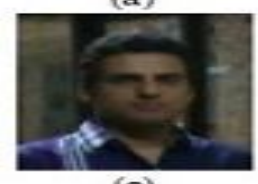

(e)

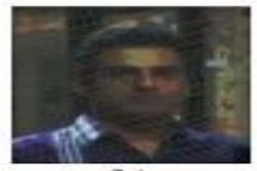

(b)

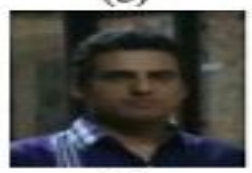

(f)

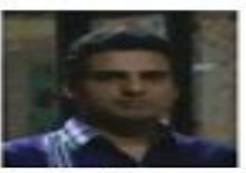

(c)

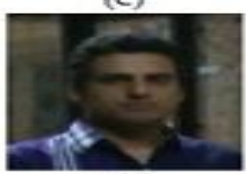

(в)

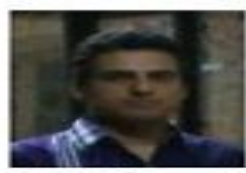

(d)

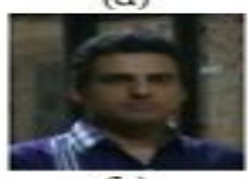

(h)

Fig 8 (a-h) $0^{\text {th }}$ to $7^{\text {th }}$ Bit Stego-images

TABLE III Result of Iqm for colour images

\begin{tabular}{|l|l|r|r|r|r|r|r|r|}
\hline IQM & $\mathbf{0}^{\text {th }} \mathbf{B i t}$ & $\mathbf{1}^{\text {st }} \mathbf{B i t}$ & $\mathbf{2}^{\text {nd }} \mathbf{B i t}$ & $\mathbf{3}^{\text {rd }} \mathbf{B i t}$ & $\mathbf{4}^{\text {th }} \mathbf{B i t}$ & $\mathbf{5}^{\text {th }} \mathbf{B i t}$ & $\mathbf{6}^{\text {th }} \mathbf{B i t}$ & $\mathbf{7}^{\text {th }} \mathbf{B i t}$ \\
\hline & 2687.5041 & 342.7982 & & & & & & \\
MSE & 6 & 9 & 50.06137 & 12.03452 & 3.43584 & 0.99917 & 0.46127 & 0.46088 \\
\hline & & & & 42.7459 & 9 & 51.4936 & 51.5100 \\
PSNR & 13.84529 & 22.56285 & 30.81184 & 37.51692 & 9 & 48.03016 & 5 & 3 \\
\hline NCC & 0.99246 & 0.99254 & 0.99259 & 0.99278 & 0.99954 & 1 & 1 & \\
\hline UIQI & 0.33915 & 0.83143 & 0.97505 & 0.99405 & 0.99829 & 0.99950 & 0.99977 & 0.99977 \\
\hline SSIM & 0.34085 & 0.83208 & 0.97514 & 0.99407 & 0.99830 & 0.999507 & 0.99977 & 0.99977 \\
\hline
\end{tabular}

TABLE IV Result of histogram comparison for colour images

\begin{tabular}{|l|l|r|r|r|r|r|r|r|}
\hline H-comp & $\mathbf{0}^{\text {th }} \mathbf{B i t}$ & $\mathbf{1}^{\text {st }} \mathbf{B i t}$ & $\mathbf{2}^{\text {nd }} \mathbf{B i t}$ & $\mathbf{3}^{\text {rd }} \mathbf{B i t}$ & $\mathbf{4}^{\text {th }} \mathbf{B i t}$ & $\mathbf{5}^{\text {th }} \mathbf{B i t}$ & $\mathbf{6}^{\text {th }} \mathbf{B i t}$ & $\mathbf{7}^{\text {th }} \mathbf{B i t}$ \\
\hline Jaccard & 0.66618 & 0.92576 & 0.98811 & 0.99711 & 0.99917 & 0.99976 & 0.99989 & 0.99989 \\
\hline Intersection & 0.99163 & 0.96342 & 0.97102 & 0.98079 & 0.98995 & 0.99455 & 0.99588 & 0.99544 \\
\hline Correlation & 0.40984 & 0.83169 & 0.97349 & 0.99466 & 0.99836 & 0.99951 & 0.99781 & 0.99985 \\
\hline Chi-square & 12.75833 & 2.89087 & 0.44306 & 0.10753 & 0.03067 & 0.00892 & 0.00412 & 0.00412 \\
\hline Bhattyacharya & 0.20944 & 0.12181 & 0.05473 & 0.02694 & 0.01448 & 0.00764 & 0.00532 & 0.00534 \\
\hline
\end{tabular}


International Journal of Computer Science \& Information Technology (IJCSIT) Vol 6, No 6, December 2014
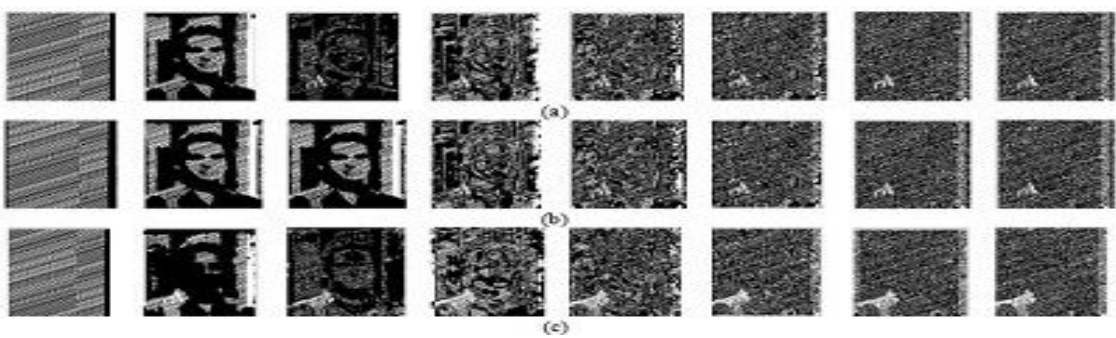

Fig 9 (a-c) RGB Bit plans of $0^{\text {th }}$ Bit Stego-image
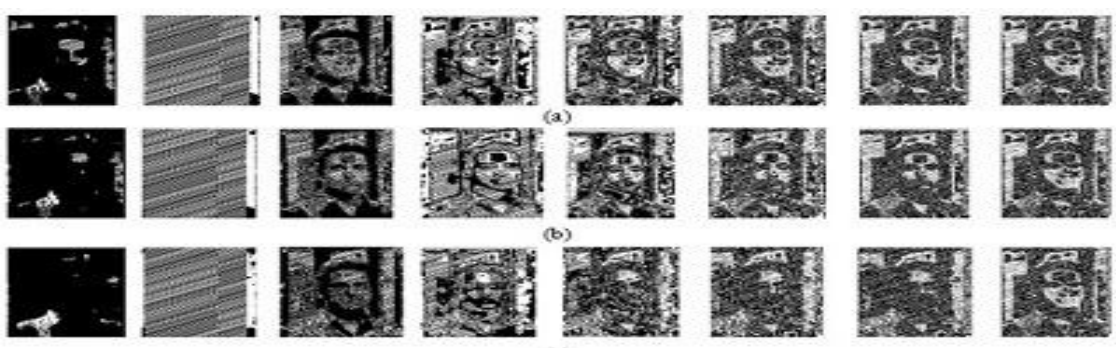

Fig 10 (a-c) RGB Bit plans of $1^{\text {st }}$ Bit Stego-image
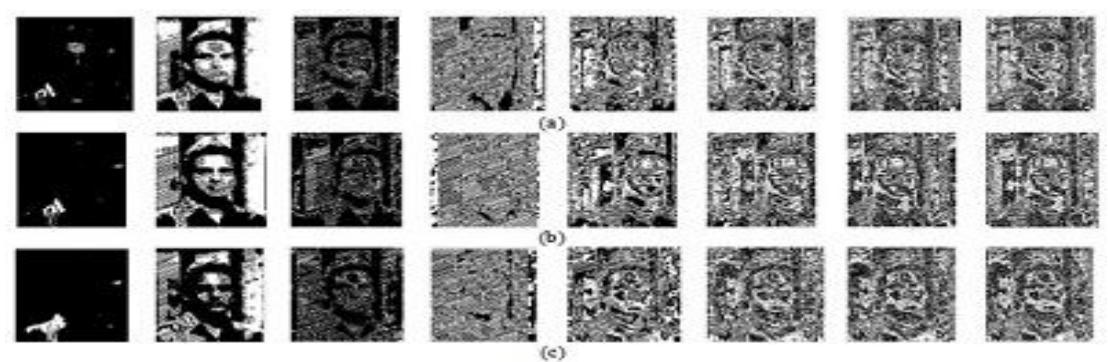

Fig 11 (a-c) RGB Bit plans of $2^{\text {nd }}$ Bit Stego-image
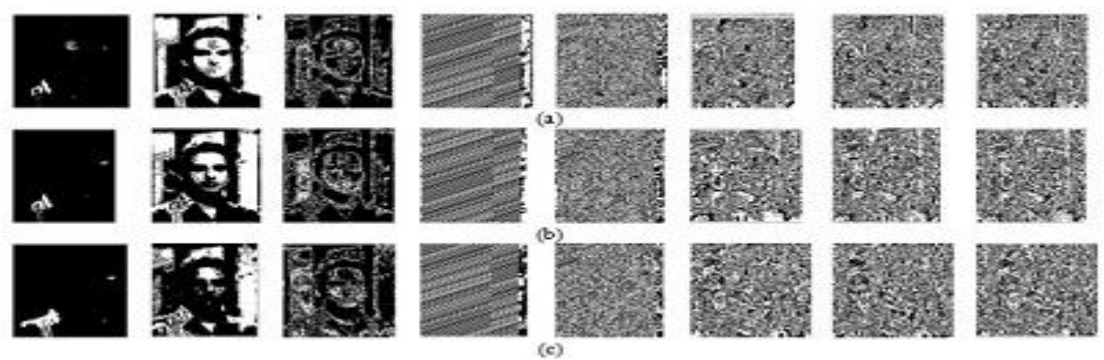

Fig 12 (a-c) RGB Bit plans of $3^{\text {rd }}$ Bit Stego-image 
International Journal of Computer Science \& Information Technology (IJCSIT) Vol 6, No 6, December 2014
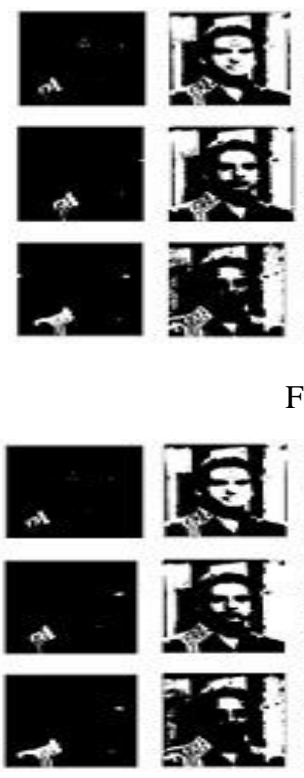
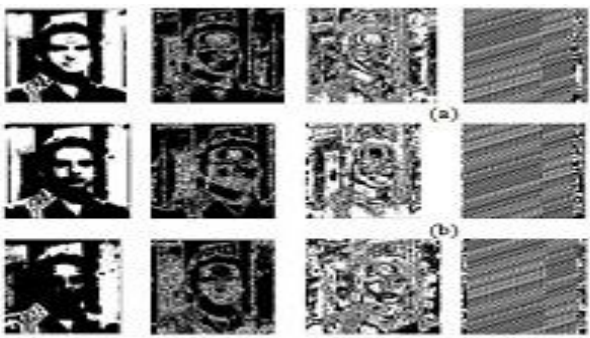
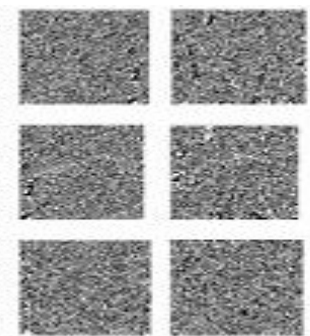

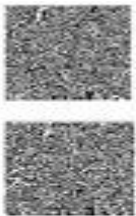

Fig 13 (a-c) RGB Bit plans of $4^{\text {th }}$ Bit Stego-image
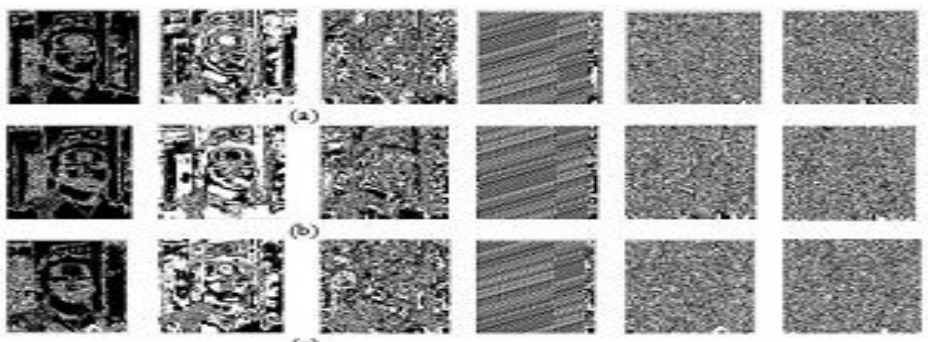

Fig 14 (a-c) RGB Bit plans of $5^{\text {th }}$ Bit Stego-image
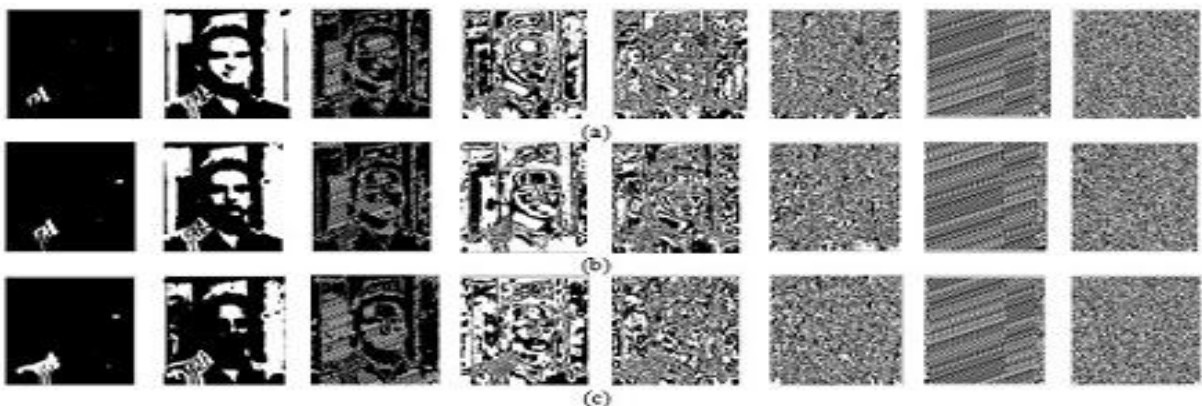

Fig 15 (a-c) RGB Bit plans of $6^{\text {th }}$ Bit Stego-image
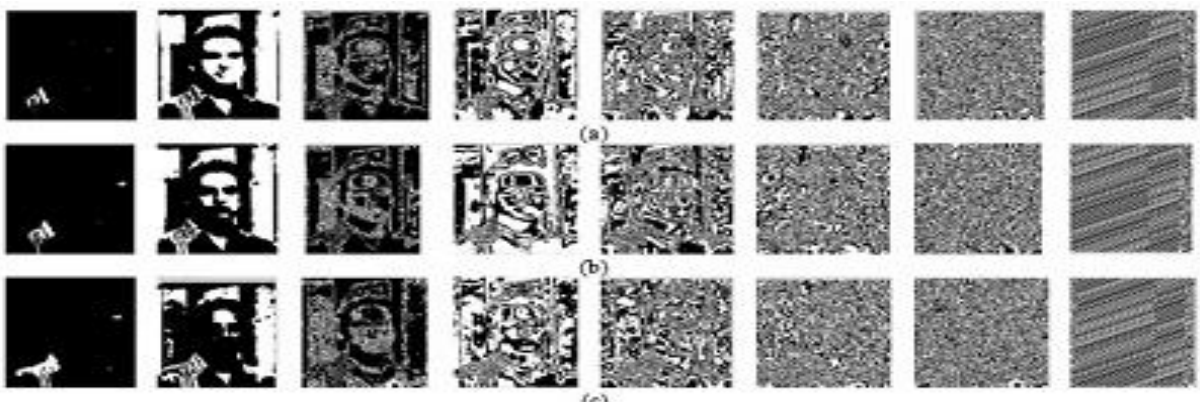

Fig 16 (a-c) RGB Bit plans of $7^{\text {th }}$ Bit Stego-image

Perceptual appearance of stego-images in first two bits $0^{\text {th }}$ bit and $2^{\text {nd }}$ bit is very poor. And change in histogram is also at great extinct for those stego-images. However improvement occurs after $4^{\text {th }}$ bit. Table I and III shows the results of MSE, PSNR, NCC, UIQI and SSIM for the greyscale and colour images. The greater the value of MSE and lesser PSNR means that perceived error is high. NCC, UIQI and SSIM closer to 1 means that perceived change in structural information is very less and 1 means identical images. And Table V shows the change 
in pixel value. This is least for $7^{\text {th }}$ bit and increases as move towards $0^{\text {th }}$ bit. All these results are the answer of the question that Why data is hidden only in the LSB of the pixel.

TABLE V Change in value of pixel after matching

\begin{tabular}{|l|l|l|}
\hline Bit Location & Maximum & Minimum \\
\hline $7^{\text {th }}(\mathrm{LSB}) \mathrm{Bit}$ & Increment/Decrement by 1 & No change \\
\hline $6^{\text {th }} \mathrm{Bit}$ & Increment/Decrement by 2 & No change \\
\hline $5^{\text {th }} \mathrm{Bit}$ & Increment/Decrement by 4 & No change \\
\hline $4^{\text {th }} \mathrm{Bit}$ & Increment/Decrement by 8 & No change \\
\hline $3^{\text {rd }} \mathrm{Bit}$ & Increment/Decrement by 16 & No change \\
\hline $2^{\text {nd }} \mathrm{Bit}$ & Increment/Decrement by 32 & No change \\
\hline $1^{\text {st }} \mathrm{Bit}$ & Increment/Decrement by 64 & No change \\
\hline $0^{\text {th }}(\mathrm{M}$ & & \\
$\mathrm{SB}) \mathrm{Bit}$ & Increment/Decrement by 128 & No change \\
\hline
\end{tabular}

\section{CONCLuSiON}

Image LSB matching steganography for every bit of pixel is critically analyzed after inserting message bits in all location of image bits. Perceptual appearance is poor in most three significant bits and remaining are good. Steganalysis is very easy for most significant bits. Perfect results of image quality methods require identical images. MSB of the image contain most important information. So change to that will give poor results of image quality measure. However as we move from MSB to LSB results becomes good. This paper also discusses the important quality evaluation methods and attacks on LSB matching. This analysis could be very helpful for those who want to work in the field of steganography.

\section{REFERENCES}

[1] N.F.Johnson, Sushil Jojadia George Mason University, "Exploring Steganography: Seeing the Unseen", (0018-916/98/\$10.00@) 1998 IEEE

[2] R.Poornima, R.J.Iswarya, “An Overview of Digital Image Steganography”, International Journal of Computer Science \& Engineering Survey (Vol.4, No 1),February 2013

[3] T.Morkel, T.H.P.Eloff, M.S.Olivier, "An Overview of Image Steganography”, ICSA Research Group, Department of Computer Science.

[4] Jammi Ashok, Y.Raju, S.Munishankaralak, K.Srinivas, Jammi Ashok, "Steganography: An Overview", et.01./International Journal of Engineering Science and Technology, (Vol.2(10)), 2010, 5985-5992

[5] Shikha Sharda, Sumit Budhiraja , "Image Steganography:A Review", International Journal of Emerging Technology and Advance Engineering (volume 3, Issue 1), January 2013

[6] V. Asha, P. Nagabhushan, N. U. Bhajantri, "Similarity Measures for Automatic Defect Detection on Patterned Textures", International Journal of Image Processing and Vision Sciences (IJIPVS) Volume-1 Issue-1, 2012

[7] Rajkumar Yadav, "Analysis of Various Image Steganography Techniques Based Upon PSNR Metric”, International Journal of P2P Network Trends and Technology-(Volume1, Issue2)- 2011, ISSN: 2249-2615

[8] M. Pavani, S. Naganjaneyulu, C. Nagaraju, " A Survey on LSB Based Steganography Methods", International Journal Of Engineering And Computer Science ISSN: 2319-7242 (Volume 2 Issue 8) August, 2013 Page No. 2464-2467

[9] Ismail Avcibas, Bulent Sankur, Khalid Sayood, "Statistical Evaluation of Image Quality Measure", Journal of Electronic Imaging, 11(2), 206-223(April 2002)

[10] Zhou Wang, Member,Hamid R. Sheikh, "Image Quality Assessment: From Error Visibility to Structural Similarity”, IEEE Transactions On Image Processing, (VOL. 13, NO. 4), APRIL 20041 
International Journal of Computer Science \& Information Technology (IJCSIT) Vol 6, No 6, December 2014

[11] Yousra A. Y. Al. Najjar, Dr. D. C. Soong, "Comparison of image quality assessment: PSNR, HVS, UIQI, SSIM”, IJSER, (Vol. 3, Issue8), August-2012. ISSN2229-5518

[12] Amhamed Saffor, Abdul Rahman Ramli, Kwan-Hoong Ng, "A Comparative Study Of Image Compression Between Jpeg And Wavelet”, Malaysian Journal of Computer Science, (Vol. 14 No. 1), June 2001, pp. 39-45. 